\title{
Energy functionals of Kirchhoff-type problems having multiple global minima
}

\section{BIAGIO RICCERI}

\begin{abstract}
In this paper, using the theory developed in [8], we obtain some results of a totally new type about a class of non-local problems. Here is a sample:

Let $\Omega \subset \mathbf{R}^{n}$ be a smooth bounded domain, with $n \geq 4$, let $a, b, \nu \in \mathbf{R}$, with $a \geq 0$ and $b>0$, and let $p \in] 0, \frac{n+2}{n-2}[$.

Then, for each $\lambda>0$ large enough and for each convex set $C \subseteq L^{2}(\Omega)$ whose closure in $L^{2}(\Omega)$ contains $H_{0}^{1}(\Omega)$, there exists $v^{*} \in C$ such that the problem

$$
\begin{cases}-\left(a+b \int_{\Omega}|\nabla u(x)|^{2} d x\right) \Delta u=\nu|u|^{p-1} u+\lambda\left(u-v^{*}(x)\right) & \text { in } \Omega \\ u=0 & \text { on } \partial \Omega\end{cases}
$$

has at least three weak solutions, two of which are global minima in $H_{0}^{1}(\Omega)$ of the corresponding energy functional.
\end{abstract}

Keywords. Kirchhoff-type problem; energy functional; global minimum; variational methods; strict minimax inequality; multiplicity.

\section{Introduction}

Here and in what follows, $\Omega \subset \mathbf{R}^{n}(n \geq 3)$ is a bounded domain with smooth boundary and $a, b \in \mathbf{R}$, with $a \geq 0$ and $b>0$.

Consider the non-local problem

$$
\begin{cases}-\left(a+b \int_{\Omega}|\nabla u(x)|^{2} d x\right) \Delta u=h(x, u) & \text { in } \Omega \\ u=0 & \text { on } \partial \Omega,\end{cases}
$$

$h: \Omega \times \mathbf{R} \rightarrow \mathbf{R}$ being a Carathéodory function.

In the past years, many papers have been produced on the existence and multiplicity of weak solutions for this problem. Usual reference papers are [1], [2], [4], [5], [6], [10].

In the present note, we are interested in the multiplicity of solutions of the above problem under the following new aspect which has not been considered before: among the solutions of the problem, at least two are global minima of the energy functional.

But, more generally, the global structure itself of the conclusions we reach is novel at all, so that no proper comparison of our results with the previous ones in the field can be made.

For instance, the following proposition summarizes very well the novelties of our main result (Theorem 1) of which it is the simplest particular case:

PROPOSITION 1. - Let $n \geq 4$, let $\nu \in \mathbf{R}$ and let $p \in] 0, \frac{n+2}{n-2}[$.

Then, for each $\lambda>0$ large enough and for each convex set $C \subseteq L^{2}(\Omega)$ whose closure in $L^{2}(\Omega)$ contains $H_{0}^{1}(\Omega)$, there exists $v^{*} \in C$ such that the problem

$$
\begin{cases}-\left(a+b \int_{\Omega}|\nabla u(x)|^{2} d x\right) \Delta u=\nu|u|^{p-1} u+\lambda\left(u-v^{*}(x)\right) & \text { in } \Omega \\ u=0 & \text { on } \partial \Omega\end{cases}
$$


has at least three solutions, two of which are global minima in $H_{0}^{1}(\Omega)$ of the functional

$$
u \rightarrow \frac{a}{2} \int_{\Omega}|\nabla u(x)|^{2} d x+\frac{b}{4}\left(\int_{\Omega}|\nabla u(x)|^{2} d x\right)^{2}-\frac{\nu}{p+1} \int_{\Omega}|u(x)|^{p+1} d x-\frac{\lambda}{2} \int_{\Omega}\left|u(x)-v^{*}(x)\right|^{2} d x .
$$

What allows us to obtain results of this totally new type is the use of theory we have recently developed in $[8]$.

\section{Results}

On the Sobolev space $H_{0}^{1}(\Omega)$, we consider the scalar product

$$
\langle u, v\rangle=\int_{\Omega} \nabla u(x) \nabla v(x) d x
$$

and the induced norm

$$
\|u\|=\left(\int_{\Omega}|\nabla u(x)|^{2} d x\right)^{\frac{1}{2}} .
$$

We denote by $\mathcal{A}$ the class of all Carathéodory functions $f: \Omega \times \mathbf{R} \rightarrow \mathbf{R}$ such that

$$
\sup _{(x, \xi) \in \Omega \times \mathbf{R}} \frac{|f(x, \xi)|}{1+|\xi|^{p}}<+\infty
$$

for some $p \in] 0, \frac{n+2}{n-2}[$.

Moreover, we denote by $\tilde{\mathcal{A}}$ the class of all Carathéodory functions $g: \Omega \times \mathbf{R} \rightarrow \mathbf{R}$ such that

$$
\sup _{(x, \xi) \in \Omega \times \mathbf{R}} \frac{|g(x, \xi)|}{1+|\xi|^{q}}<+\infty
$$

for some $q \in] 0, \frac{2}{n-2}[$.

Furthermore, we denote by $\hat{\mathcal{A}}$ the class of all functions $h: \Omega \times \mathbf{R} \rightarrow \mathbf{R}$ of the type

$$
h(x, \xi)=f(x, \xi)+\alpha(x) g(x, \xi)
$$

with $f \in \mathcal{A}, g \in \tilde{\mathcal{A}}$ and $\alpha \in L^{2}(\Omega)$. For each $h \in \hat{\mathcal{A}}$, we define the functional $I_{h}: H_{0}^{1}(\Omega) \rightarrow \mathbf{R}$, by putting

$$
I_{h}(u)=\int_{\Omega} H(x, u(x)) d x
$$

for all $u \in H_{0}^{1}(\Omega)$, where

$$
H(x, \xi)=\int_{0}^{\xi} h(x, t) d t
$$

for all $(x, \xi) \in \Omega \times \mathbf{R}$.

By classical results (involving the Sobolev embedding theorem), the functional $I_{h}$ turns out to be sequentially weakly continuous, of class $C^{1}$, with compact derivative given by

$$
I_{h}^{\prime}(u)(w)=\int_{\Omega} h(x, u(x)) w(x) d x
$$

for all $u, w \in H_{0}^{1}(\Omega)$. 
Now, let us recall that, given $h \in \hat{\mathcal{A}}$, a weak solution of the problem

$$
\begin{cases}-\left(a+b \int_{\Omega}|\nabla u(x)|^{2} d x\right) \Delta u=h(x, u) & \text { in } \Omega \\ u=0 & \text { on } \partial \Omega\end{cases}
$$

is any $u \in H_{0}^{1}(\Omega)$ such that

$$
\left(a+b \int_{\Omega}|\nabla u(x)|^{2} d x\right) \int_{\Omega} \nabla u(x) \nabla w(x) d x=\int_{\Omega} h(x, u(x)) w(x) d x
$$

for all $w \in H_{0}^{1}(\Omega)$. Let $\Phi: H_{0}^{1}(\Omega) \rightarrow \mathbf{R}$ be the functional defined by

$$
\Phi(u)=\frac{a}{2}\|u\|^{2}+\frac{b}{4}\|u\|^{4}
$$

for all $u \in H_{0}^{1}(\Omega)$.

Hence, the weak solutions of the problem are precisely the critical points in $H_{0}^{1}(\Omega)$ of the functional $\Phi-I_{h}$ which is said to be the energy functional of the problem.

Here is our main result:

THEOREM 1. - Let $n \geq 4$, let $f \in \mathcal{A}$ and let $g \in \tilde{\mathcal{A}}$ be such that the set

$$
\left\{x \in \Omega: \sup _{\xi \in \mathbf{R}}|g(x, \xi)|>0\right\}
$$

has a positive measure.

Then, there exist $\lambda^{*} \geq 0$ such that, for each $\lambda>\lambda^{*}$ and for each convex set $C \subseteq L^{2}(\Omega)$ whose closure in $L^{2}(\Omega)$ contains the set $\left\{G(\cdot, u(\cdot)): u \in H_{0}^{1}(\Omega)\right\}$, there exists $v^{*} \in C$ such that the problem

$$
\begin{cases}-\left(a+b \int_{\Omega}|\nabla u(x)|^{2} d x\right) \Delta u=f(x, u)+\lambda\left(G(x, u)-v^{*}(x)\right) g(x, u) & \text { in } \Omega \\ u=0 & \text { on } \partial \Omega\end{cases}
$$

has at least three weak solutions, two of which are global minima in $H_{0}^{1}(\Omega)$ of the functional

$$
u \rightarrow \frac{a}{2} \int_{\Omega}|\nabla u(x)|^{2} d x+\frac{b}{4}\left(\int_{\Omega}|\nabla u(x)|^{2} d x\right)^{2}-\int_{\Omega} F(x, u(x)) d x-\frac{\lambda}{2} \int_{\Omega}\left|G(x, u(x))-v^{*}(x)\right|^{2} d x .
$$

If, in addition, the functional

$$
u \rightarrow \frac{a}{2} \int_{\Omega}|\nabla u(x)|^{2} d x+\frac{b}{4}\left(\int_{\Omega}|\nabla u(x)|^{2} d x\right)^{2}-\int_{\Omega} F(x, u(x)) d x
$$

has at least two global minima in $H_{0}^{1}(\Omega)$ and the function $G(x, \cdot)$ is strictly monotone for all $x \in \Omega$, then $\lambda^{*}=0$.

The main tool we use to prove Theorem 1 is Theorem $\mathrm{C}$ below which is, in turn, a direct consequence of two other results recently established in [8].

To state Theorem $\mathrm{C}$ in a compact form, we now introduce some notations.

Here and in what follows, $X$ is a non-empty set, $V, Y$ are two topological spaces, $y_{0}$ is a point in $Y$.

We denote by $\mathcal{G}$ the family of all lower semicontinuous functions $\varphi: Y \rightarrow\left[0,+\infty\left[\right.\right.$, with $\varphi^{-1}(0)=\left\{y_{0}\right\}$, such that, for each neighbourhood $U$ of $y_{0}$, one has

$$
\inf _{Y \backslash U} \varphi>0 .
$$


Moreover, we denote by $\mathcal{H}$ the family of all functions $\Psi: X \times V \rightarrow Y$ such that, for each $x \in X, \Psi(x, \cdot)$ is continuous, injective, open, takes the value $y_{0}$ at a point $v_{x}$ and the function $x \rightarrow v_{x}$ is not constant. Furthermore, we denote by $\mathcal{M}$ the family of all functions $J: X \rightarrow \mathbf{R}$ whose set of all global minima (noted by $M_{J}$ ) is non-empty.

Finally, for each $\varphi \in \mathcal{G}, \Psi \in \mathcal{H}$ and $J \in \mathcal{M}$, we put

$$
\theta(\varphi, \Psi, J)=\inf \left\{\frac{J(x)-J(u)}{\varphi\left(\Psi\left(x, v_{u}\right)\right)}:(u, x) \in M_{J} \times X \text { with } v_{x} \neq v_{u}\right\} .
$$

When $X$ is a topological space, a function $\psi: X \rightarrow \mathbf{R}$ is said to be inf-compact if $\left.\left.\psi^{-1}(]-\infty, r\right]\right)$ is compact for all $r \in \mathbf{R}$.

THEOREM A ([8], Theorem 3.1). - Let $\varphi \in \mathcal{G}, \Psi \in \mathcal{H}$ and $J \in \mathcal{M}$.

Then, for each $\lambda>\theta(\varphi, \Psi, J)$, one has

$$
\sup _{v \in V} \inf _{x \in X}(J(x)-\lambda \varphi(\Psi(x, v)))<\inf _{x \in X} \sup _{z \in X}\left(J(x)-\lambda \varphi\left(\Psi\left(x, v_{z}\right)\right)\right) .
$$

THEOREM B ([8], Theorem 3.2). - Let $X$ be a topological space, E a real Hausdorff topological vector space, $C \subseteq E$ a convex set, $f: X \times C \rightarrow \mathbf{R}$ a function which is lower semicontinuous and inf-compact in $X$, and upper semicontinuous and concave in $C$. Assume also that

$$
\sup _{v \in C} \inf _{x \in X} f(x, v)<\inf _{x \in X} \sup _{v \in C} f(x, v) .
$$

Then, there exists $v^{*} \in C$ such that the function $f\left(\cdot, v^{*}\right)$ has at least two global minima in $X$.

THEOREM C. - Let $\varphi \in \mathcal{G}, \Psi \in \mathcal{H}$ and $J \in \mathcal{M}$. Moreover, assume that $X$ is a topological space, that $V$ is a real Hausdorff topological vector space and that $\varphi(\Psi(x, \cdot))$ is convex and continuous for each $x \in X$. Finally, let $\lambda>\theta(\varphi, \Psi, J)$ and let $C \subseteq V$ be a convex set, with $\left\{v_{x}: x \in X\right\} \subseteq \bar{C}$, such that the function $x \rightarrow J(x)-\lambda \varphi(\Psi(x, v))$ is lower semicontinuous and inf-compact in $X$ for all $v \in C$.

Under such hypotheses, there exists $v^{*} \in C$ such that the function $x \rightarrow J(x)-\lambda \varphi\left(\Psi\left(x, v^{*}\right)\right)$ has at least two global minima in $X$.

PROOF. Set

$$
D=\left\{v_{x}: x \in X\right\}
$$

and, for each $(x, v) \in X \times V$, put

$$
f(x, v)=J(x)-\lambda \varphi(\Psi(x, v))
$$

Theorem A ensures that

$$
\sup _{v \in V} \inf _{x \in X} f(x, v)<\inf _{x \in X} \sup _{v \in D} f(x, v) .
$$

But, since $f(x, \cdot)$ is continuous and $D \subseteq \bar{C}$, we have

$$
\sup _{v \in D} f(x, v)=\sup _{v \in \bar{D}} f(x, v) \leq \sup _{v \in \bar{C}} f(x, v)=\sup _{v \in C} f(x, v)
$$

for all $x \in X$, and hence, taking (3) into account, it follows that

$$
\sup _{v \in C} \inf _{x \in X} f(x, v)<\inf _{x \in X} \sup _{v \in D} f(x, v) \leq \inf _{x \in X} \sup _{v \in C} f(x, v) .
$$

At this point, the conclusion follows applying Theorem B to the restriction of the function $f$ to $X \times C$.

Proof of Theorem 1. For each $\lambda \geq 0, v \in L^{2}(\Omega)$, consider the function $h_{\lambda, v}: \Omega \times \mathbf{R} \rightarrow \mathbf{R}$ defined by

$$
h_{\lambda, v}(x, \xi)=f(x, \xi)+\lambda(G(x, \xi)-v(x)) g(x, \xi)
$$


for all $(x, \xi) \in \Omega \times \mathbf{R}$. Clearly, the function $h_{\lambda, v}$ lies in $\hat{\mathcal{A}}$ and

$$
H_{\lambda, v}(x, \xi)=F(x, \xi)+\frac{\lambda}{2}\left(|G(x, \xi)-v(x)|^{2}-|v(x)|^{2}\right) .
$$

Moreover, if $p \in] 0, \frac{n+2}{n-2}[$ and $q \in] 0, \frac{2}{n-2}\left[\right.$ are such that (1) and (2) hold, for some constant $c_{\lambda, v}$, we have

$$
\int_{\Omega}\left|H_{\lambda, v}(x, u(x))\right| d x \leq c_{\lambda, v}\left(\int_{\Omega}|u(x)|^{p+1} d x+\int_{\Omega}|u(x)|^{2(q+1)} d x+1\right)
$$

for all $u \in H_{0}^{1}(\Omega)$. Therefore, by the Sobolev embedding theorem, for a constant $\tilde{c}_{\lambda, v}$, we have

$$
\Phi(u)-I_{h_{\lambda, v}}(u) \geq \frac{b}{4}\|u\|^{4}-\tilde{c}_{\lambda, v}\left(\|u\|^{p+1}+\|u\|^{2(q+1)}+1\right)
$$

for all $u \in H_{0}^{1}(\Omega)$. On the other hand, since $n \geq 4$, one has

$$
\max \{p+1,2(q+1)\}<\frac{2 n}{n-2} \leq 4 .
$$

Consequently, from (4), we infer that

$$
\lim _{\|u\| \rightarrow+\infty}\left(\Phi(u)-I_{h_{\lambda, v}}(u)\right)=+\infty
$$

Since the functional $\Phi-I_{h_{\lambda, v}}$ is sequentially weakly lower semicontinuous, by the Eberlein-Smulyan theorem and by (5), it follows that it is inf-weakly compact.

Now, we are going to apply Theorem $\mathrm{C}$ taking $X=H_{0}^{1}(\Omega)$ with the weak topology, $V=Y=L^{2}(\Omega)$ with the strong topology and $y_{0}=0$. Also, we take

$$
\varphi(w)=\frac{1}{2} \int_{\Omega}|w(x)|^{2} d x
$$

for all $w \in L^{2}(\Omega)$. Clearly, $\varphi \in \mathcal{G}$. Furthermore, we take

$$
\Psi(u, v)(x)=G(x, u(x))-v(x)
$$

for all $u \in H_{0}^{1}(\Omega), v \in L^{2}(\Omega), x \in \Omega$. Clearly, $\Psi(u, v) \in L^{2}(\Omega), \Psi(u, \cdot)$ is a homeomorphism, and we have

$$
v_{u}(x)=G(x, u(x)) .
$$

We now show that the map $u \rightarrow v_{u}$ is not constant in $H_{0}^{1}(\Omega)$. Set

$$
A=\left\{x \in \Omega: \sup _{\xi \in \mathbf{R}}|g(x, \xi)|>0\right\} .
$$

By assumption, meas $(A)>0$. Then, by the classical Scorza-Dragoni theorem ([3], Theorem 2.5.19), there exists a compact set $K \subset A$, of positive measure, such that the restriction of $G$ to $K \times \mathbf{R}$ is continuous. Fix a point $\tilde{x} \in K$ such that the intersection of $K$ and any ball centered at $\tilde{x}$ has a positive measure. Of course, the function $G(\tilde{x}, \cdot)$ is not constant. Fix $\xi_{1}, \xi_{2} \in \mathbf{R}$ such that

$$
G\left(\tilde{x}, \xi_{1}\right)<G\left(\tilde{x}, \xi_{2}\right) .
$$

By continuity, there is a closed ball $B(\tilde{x}, r)$ such that

$$
G\left(x, \xi_{1}\right)<G\left(x, \xi_{2}\right)
$$


for all $x \in K \cap B(\tilde{x}, r)$. Finally, consider two functions $u_{1}, u_{2} \in H_{0}^{1}(\Omega)$ such that

$$
u_{1}(x)=\xi_{1}
$$

and

$$
u_{2}(x)=\xi_{2}
$$

for all $x \in K \cap B(\tilde{x}, r)$. So

$$
G\left(x, u_{1}(x)\right)<G\left(x, u_{2}(x)\right)
$$

for all $x \in K \cap B(\tilde{x}, r)$. Hence, as meas $(K \cap B(\tilde{x}, r))>0$, we infer that $v_{u_{1}} \neq v_{u_{2}}$, as claimed. As a consequence, $\Psi \in \mathcal{H}$. Of course, $\varphi(\Psi(u, \cdot))$ is continuous and convex for all $u \in X$. Finally, take

$$
J=\Phi-I_{f}
$$

Clearly, $J \in \mathcal{M}$. So, for what seen above, all the assumptions of Theorem C are satisfied. Consequently, if we take

$$
\lambda^{*}=\theta(\varphi, \Psi, J)
$$

and fix $\lambda>\lambda^{*}$ and a convex set $C \subseteq L^{2}(\Omega)$ whose closure in $L^{2}(\Omega)$ contains the set $\left\{G(\cdot, u(\cdot)): u \in H_{0}^{1}(\Omega)\right\}$, there exists $v^{*} \in C$ such that the functional $\Phi-I_{h_{\lambda, v^{*}}}$ has at least two global minima in $H_{0}^{1}(\Omega)$ which are, therefore, weak solutions of the problem we are dealing with. To guarantee the existence of a third solution, denote by $k$ the inverse of the restriction of the function $a t+b t^{3}$ to $[0,+\infty[$. Let $T: X \rightarrow X$ be the operator defined by

$$
T(w)= \begin{cases}\frac{k(\|w\|)}{\|w\|} w & \text { if } w \neq 0 \\ 0 & \text { if } w=0,\end{cases}
$$

Since $k$ is continuous and $k(0)=0$, the operator $T$ is continuous in $X$. For each $u \in X \backslash\{0\}$, we have

$$
T\left(\Phi^{\prime}(u)\right)=T\left(\left(a+b\|u\|^{2}\right) u\right)=\frac{k\left(\left(a+b\|u\|^{2}\right)\|u\|\right)}{\left(a+b\|u\|^{2}\right)\|u\|}\left(a+b\|u\|^{2}\right) u=\frac{\|u\|}{\left(a+b\|u\|^{2}\right)\|u\|}\left(a+b\|u\|^{2}\right) u=u .
$$

In other words, $T$ is a continuous inverse of $\Phi^{\prime}$. Then, since $I_{h_{\lambda, v^{*}}}^{\prime}$ is compact, the functional $\Phi-I_{h_{\lambda, v^{*}}}$ satisfies the Palais-Smale condition ([9], Example 38.25) and hence the existence of a third critical point of the same functional is assured by Corollary 1 of [7].

Finally, assume that the functional $\Phi-I_{f}$ has at least two global minima, say $\hat{u}_{1}, \hat{u}_{2}$. So, the set

$$
\Gamma:=\left\{x \in \Omega: \hat{u}_{1}(x) \neq \hat{u}_{2}(x)\right\}
$$

has a positive measure. By assumption, we have

$$
G\left(x, \hat{u}_{1}(x)\right) \neq G\left(x, \hat{u}_{2}(x)\right)
$$

for all $x \in \Gamma$, and hence $v_{\hat{u}_{1}} \neq v_{\hat{u}_{2}}$. Then, by definition, we have

$$
0 \leq \theta(\varphi, \Psi, J) \leq \frac{J\left(\hat{u}_{1}\right)-J\left(\hat{u}_{2}\right)}{\varphi\left(\Psi\left(\hat{u}_{1}, v_{\hat{u}_{2}}\right)\right)}=0
$$

and so $\lambda^{*}=0$ in view of $(6)$.

REMARK 1. - In Theorem 1, the assumption made on $g$ (besides $g \in \tilde{\mathcal{A}}$ ) is essential. Indeed, if $g(x, \xi)=0$ for almost every $x \in \Omega$ and for every $\xi \in \mathbf{R}$, then, taking $f=0$ (which is an allowed choice), the problem would have the zero solution only.

REMARK 2. - The assumption $n \geq 4$ is likewise essential. Indeed, Proposition 1 (which comes from Theorem 1 taking $f(x, \xi)=\nu|\xi|^{p-1} \xi$ and $g(x, \xi)=1$ ) does not hold if $n=3$ and $\nu>0$. To see this, take 
$p=4$ (which is compatible with the condition $p<\frac{n+2}{n-2}$ when $n=3$ ) and observe that the corresponding energy functional is unbounded below.

Besides Proposition 1, among the consequences of Theorem 1, we highlight the following

THEOREM 2. - Let $n \geq 4$, let $f \in \mathcal{A}$ and let $g \in \tilde{\mathcal{A}}$ be such the set

$$
\left\{x \in \Omega: \sup _{\xi \in \mathbf{R}} F(x, \xi)>0\right\}
$$

has a positive measure. Moreover, assume that, for each $x \in \Omega, f(x, \cdot)$ is odd, $g(x, \cdot)$ is even and $G(x, \cdot)$ is strictly monotone.

Then, for every $\lambda>0$, there exists $\mu^{*}>0$ such that, for each $\mu>\mu^{*}$ and for each convex set $C \subseteq L^{2}(\Omega)$ whose closure in $L^{2}(\Omega)$ contains the set $\left\{G(\cdot, u(\cdot)): u \in H_{0}^{1}(\Omega)\right\}$, there exists $v^{*} \in C$ such that the problem

$$
\begin{cases}-\left(a+b \int_{\Omega}|\nabla u(x)|^{2} d x\right) \Delta u=\mu f(x, u)-\lambda v^{*}(x) g(x, u) & \text { in } \Omega \\ u=0 & \text { on } \partial \Omega\end{cases}
$$

has at least three weak solutions, two of which are global minima in $H_{0}^{1}(\Omega)$ of the functional

$$
u \rightarrow \frac{a}{2} \int_{\Omega}|\nabla u(x)|^{2} d x+\frac{b}{4}\left(\int_{\Omega}|\nabla u(x)|^{2} d x\right)^{2}-\mu \int_{\Omega} F(x, u(x)) d x+\lambda \int_{\Omega} v^{*}(x) G(x, u(x)) d x .
$$

PROOF. Set

$$
D=\left\{x \in \Omega: \sup _{\xi \in \mathbf{R}} F(x, \xi)>0\right\} .
$$

By assumption, meas $(D)>0$. Then, by the Scorza-Dragoni theorem, there exists a compact set $K \subset D$, of positive measure, such that the restriction of $F$ to $K \times \mathbf{R}$ is continuous. Fix a point $\hat{x} \in K$ such that the intersection of $K$ and any ball centered at $\hat{x}$ has a positive measure. Choose $\hat{\xi} \in \mathbf{R}$ so that $F(\hat{x}, \hat{\xi})>0$. By continuity, there is $r>0$ such that

$$
F(x, \hat{\xi})>0
$$

for all $x \in K \cap B(\hat{x}, r)$. Set

$$
M=\sup _{(x, \xi) \in \Omega \times[-|\hat{\xi}|,|\hat{\xi}|]}|F(x, \xi)| .
$$

Since $f \in \mathcal{A}$, we have $M<+\infty$. Next, choose an open set $\tilde{\Omega}$ such that

$$
K \cap B(\hat{x}, r) \subset \tilde{\Omega} \subset \Omega
$$

and

$$
\operatorname{meas}(\tilde{\Omega} \backslash(K \cap B(\hat{x}, r)))<\frac{\int_{K \cap B(\hat{x}, r)} F(x, \hat{\xi}) d x}{M} .
$$

Finally, choose a function $\tilde{u} \in H_{0}^{1}(\Omega)$ such that

$$
\tilde{u}(x)=\hat{\xi}
$$

for all $x \in K \cap B(x, r)$,

$$
\tilde{u}(x)=0
$$

for all $x \in \Omega \backslash \tilde{\Omega}$ and

$$
|\tilde{u}(x)| \leq|\hat{\xi}|
$$


for all $x \in \Omega$. Thus, we have

$$
\begin{gathered}
\int_{\Omega} F(x, \tilde{u}(x)) d x=\int_{K \cap B(\hat{x}, r)} F(x, \hat{\xi}) d x+\int_{\tilde{\Omega} \backslash(K \cap B(\hat{x}, r))} F(x, \tilde{u}(x)) d x \\
>\int_{K \cap B(\hat{x}, r)} F(x, \hat{\xi}) d x-M \operatorname{meas}(\tilde{\Omega} \backslash(K \cap B(\hat{x}, r)))>0 .
\end{gathered}
$$

Now, fix any $\lambda>0$ and set

$$
\mu^{*}=\frac{\Phi(\tilde{u})+\frac{\lambda}{2} I_{G g}(\tilde{u})}{I_{f}(\tilde{u})} .
$$

Fix $\mu>\mu^{*}$. Hence

$$
\Phi(\tilde{u})-\mu I_{f}(\tilde{u})+\frac{\lambda}{2} I_{G g}(\tilde{u})<0 .
$$

From this, we infer that the functional $\Phi-\mu I_{f}+\frac{\lambda}{2} I_{G g}$ possesses at least to global minima since it is even. At this point, we can apply Theorem 1 to the functions $g$ and $\mu f-\lambda G g$. Our current conclusion follows from the one of Theorem 1 since we have $\lambda^{*}=0$ and hence we can take the same fixed $\lambda>0$.

We conclude by proposing two very challenging problems assuming $n \geq 4$.

PROBLEM 1. - Does Proposition 1 hold if $p=\frac{n+2}{n-2}$ ?

PROBLEM 2. - Keeping the assumptions on $f$, does Theorem 2 hold for any $g \in \tilde{\mathcal{A}}$ ?

Acknowledgement. The author has been supported by the Gruppo Nazionale per l'Analisi Matematica, la Probabilità e le loro Applicazioni (GNAMPA) of the Istituto Nazionale di Alta Matematica (INdAM). 


\section{References}

[1] C. O. ALVES, F. S. J. A. CORRÊA and T. F. MA, Positive solutions for a quasilinear elliptic equations of Kirchhoff type, Comput. Math. Appl., 49 (2005), 85-93.

[2] M. CHIPOT and B. LOVAT, Some remarks on non local elliptic and parabolic problems, Nonlinear Anal., 30 (1997), 4619-4627.

[3] Z. DENKOWSKI, S. MIGÓRSKI and N. S. PAPAGEORGIOU, An Introduction to Nonlinear Analysis: Theory, Kluwer Academic Publishers, 2003.

[4] X. HE and W. ZOU, Infinitely many positive solutions for Kirchhoff-type problems, Nonlinear Anal., 70 (2009), 1407-1414.

[5] A. MAO and Z. ZHANG, Sign-changing and multiple solutions of Kirchhoff type problems without the P. S. condition, Nonlinear Anal., 70 (2009), 1275-1287.

[6] K. PERERA and Z. T. ZHANG, Nontrivial solutions of Kirchhoff-type problems via the Yang index, J. Differential Equations, 221 (2006), 246-255.

[7] P. PUCCI and J. SERRIN, A mountain pass theorem, J. Differential Equations, 60 (1985), 142-149.

[8] B. RICCERI, A strict minimax inequality criterion and some of its consequences, Positivity, 16 (2012), 455-470.

[9] E. ZEIDLER, Nonlinear functional analysis and its applications, vol. III, Springer-Verlag, 1985.

[10] Z. T. ZHANG and K. PERERA, Sign changing solutions of Kirchhoff type problems via invariant sets of descent flow, J. Math. Anal. Appl., 317 (2006), 456-463.

Department of Mathematics

University of Catania

Viale A. Doria 6

95125 Catania

Italy e-mail address: ricceri@dmi.unict.it 